\title{
New Filtering Algorithms for Combinations of Among Constraints
}

\author{
Willem-Jan van Hoeve · Gilles Pesant . \\ Louis-Martin Rousseau · Ashish Sabharwal
}

Received: date / Accepted: date

\begin{abstract}
Several combinatorial problems, such as car sequencing and rostering, feature sequence constraints, restricting the number of occurrences of certain values in every subsequence of a given length. We present three new filtering algorithms for the sequence constraint, including the first that establishes domain consistency in polynomial time. The filtering algorithms have complementary strengths: One borrows ideas from dynamic programming; another reformulates it as a regular constraint; the last is customized. The last two algorithms establish domain consistency, and the customized one does so in polynomial time. We provide experimental results that demonstrate the practical usefulness of each. We also show that the customized algorithm applies naturally to a generalized version of the sequence constraint that allows subsequences of varied lengths. The significant computational advantage of using a single generalized sequence constraint over a semantically equivalent collection of among or sequence constraints is demonstrated empirically.
\end{abstract}

Keywords sequence constraint · domain consistency $\cdot$ polynomial time filtering · car sequencing $\cdot$ regular

W.-J. van Hoeve

Tepper School of Business, Carnegie Mellon University, Pittsburgh, PA 15213, U.S.A.

Former address: Dept. of Computer Science, Cornell University, Ithaca, NY 14853, U.S.A.

E-mail:vanhoeve@andrew.cmu.edu

G. Pesant · L.-M. Rousseau

École Polytechnique de Montréal, Montreal, Canada

Centre for Research on Transportation (CRT), Université de Montréal, Montreal, H3C 3J7, Canada

E-mail: \{pesant,louism\}@ crt.umontreal.ca

L.-M. Rousseau

Oméga Optimisation Inc.

A. Sabharwal

Dept. of Computer Science, Cornell University, Ithaca, NY 14853, U.S.A.

E-mail: sabhar@cs.cornell.edu 


\section{Introduction}

The sequence constraint appears in several combinatorial problems such as car manufacturing and rostering. It can be regarded as a collection of among constraints that must hold simultaneously. An among constraint restricts the number of variables that can be assigned a value from a specific subset of domain values. For example, consider a nurse-rostering problem in which each nurse can work at most 2 night shifts during every 7 consecutive days. The among constraint specifies the 2-out-of-7 restriction, while the sequence constraint imposes such a constraint for every subsequence of 7 days.

The sequence constraint has been a topic of study in the constraint programming community since 1988 , when the car sequencing problem was first introduced [7]. Initially, the various among constraints underlying the sequence constraint were treated individually. Beldiceanu and Contejean [3] first proposed to view them together as one global sequence constraint. The constraint is also referred to as among_seq [4].

Beldiceanu and Carlsson [2] proposed a filtering algorithm for the sequence constraint, while Régin and Puget [13] presented a filtering algorithm for the sequence constraint in combination with a global cardinality constraint [12] for a car sequencing application. Neither approach, however, establishes domain consistency. As the constraint is inherent in many real-life problems, improved filtering can have a substantial industrial impact.

In this work we present three novel filtering algorithms for the sequence constraint. The first is based on dynamic programming concepts and runs in polynomial time, but does not establish domain consistency. The second algorithm is based on the regular constraint [10] and establishes domain consistency. It needs exponential time in the worst case, but in many practical cases it is very efficient. Our third algorithm establishes domain consistency in polynomial time and is the first to do so. It can be applied to a generalized version of the sequence constraint in which the subsequences considered may be of varied length. Moreover the number of occurrences may also vary per subsequence. Each algorithm has advantages over the others, either in terms of (asymptotic) running time or in terms of filtering.

Our experimental results demonstrate that our newly proposed algorithms significantly improve the state of the art. On individual sequence constraints, these algorithms are much faster than the standard (partial) filtering implementations available in the Ilog Solver library; they often reduce the number of backtracks from over a hundred thousand to zero or near-zero. On the car sequencing problem benchmark, these algorithms are able to solve more instances or achieve substantial speed-up (either on their own or as a redundant constraint added to the Ilog sequence constraint). Finally, when certain more complex combinations of among constraints are present, such as in the rostering example above, our generalized sequence constraint implementation is able to treat them all as a single global constraint, and reduces the filtering time from around half an hour to just a few seconds.

The rest of the article is structured as follows. Section 2.1 presents some background and notation on constraint programming, while Section 2.2 recalls and discusses the among and sequence constraints. Sections 3, 4, and 5 describe our three 
new filtering algorithms for the sequence constraint. Section 6 compares the algorithms experimentally. Finally, Section 7 summarizes the contributions of the work and discusses possible extensions.

\section{Background}

We first introduce basic constraint programming concepts and then discuss in detail the two constraints of interest: among and sequence. For more information on constraint programming we refer the reader to [1] and [5].

\subsection{Constraint Programming Preliminaries}

Let $x$ be a variable. The domain of $x$ is the set of values that can be assigned to $x$ and is denoted by $D(x)$. In this work we only consider variables with finite domains. Let $X=x_{1}, x_{2}, \ldots, x_{k}$ be a sequence of variables. We denote $D(X)=\bigcup_{1 \leq i \leq k} D\left(x_{i}\right)$. A constraint $C$ on $X$ is defined as a subset of the Cartesian product of the domains of the variables in $X$, i.e. $C \subseteq D\left(x_{1}\right) \times D\left(x_{2}\right) \times \cdots \times D\left(x_{k}\right)$. A tuple $\left(d_{1}, \ldots, d_{k}\right) \in C$ is called a solution to $C$. We also say that the tuple satisfies $C$. A value $d \in D\left(x_{i}\right)$ for some $i=1, \ldots, k$ is inconsistent with respect to $C$ if it does not belong to a tuple of $C$, otherwise it is consistent. $C$ is inconsistent if it does not contain a solution. Otherwise, $C$ is called consistent.

A constraint satisfaction problem, or a CSP, is defined by a finite sequence of variables $\mathscr{X}=x_{1}, x_{2}, \ldots, x_{n}$, together with a finite set of constraints $\mathscr{C}$, each on a subsequence of $\mathscr{X}$. The goal is to find an assignment $x_{i}=d_{i}$ with $d_{i} \in D\left(x_{i}\right)$ for $i=1, \ldots, n$, such that all constraints are satisfied. This assignment is called a solution to the CSP.

The solution process of constraint programming interleaves constraint propagation, or propagation in short, and search. The search process essentially consists of enumerating all possible variable-value combinations, until we find a solution or prove that none exists. We say that this process constructs a search tree. To reduce the exponential number of combinations, constraint propagation is applied to each node of the search tree: Given the current domains and a constraint $C$, remove domain values that do not belong to a solution to $C$. This is repeated for all constraints until no more domain value can be removed. The removal of inconsistent domain values is called filtering.

In order to be effective, filtering algorithms should be efficient, because they are applied many times during the solution process. Further, they should remove as many inconsistent values as possible. If a filtering algorithm for a constraint $C$ removes all inconsistent values from the domains with respect to $C$, we say that it makes $C$ domain consistent. Formally:

Definition 1 (Domain consistency, [9]) A constraint $C$ on variables $x_{1}, \ldots, x_{k}$ is called domain consistent if for each variable $x_{i}$ and each value $d_{i} \in D\left(x_{i}\right)(i=$ $1, \ldots, k)$, there exist a value $d_{j} \in D\left(x_{j}\right)$ for all $j \neq i$ such that $\left(d_{1}, \ldots, d_{k}\right) \in C$. 
In the literature, domain consistency is also referred to as hyper-arc consistency or generalized-arc consistency.

Establishing domain consistency for binary constraints (constraints defined on two variables) is inexpensive. For higher arity constraints this is not necessarily the case since the naïve approach requires time that is exponential in the number of variables. Nevertheless the underlying structure of a constraint can sometimes be exploited to establish domain consistency much more efficiently.

\subsection{The Among and Sequence Constraints}

The among constraint restricts the number of variables that can be assigned a value from a specific subset of domain values:

Definition 2 (Among constraint, [3]) Let $X=x_{1}, x_{2}, \ldots, x_{q}$ be a sequence of variables and let $S$ be a set of domain values. Let $0 \leq \ell \leq u \leq q$ be constants. Then

$$
\begin{aligned}
\operatorname{among}(X, S, \ell, u)=\left\{\left(d_{1}, \ldots, d_{q}\right) \mid\right. & \forall i \in\{1, \ldots, q\} d_{i} \in D\left(x_{i}\right), \\
& \left.\quad \leq\left|\left\{i \in\{1, \ldots, q\}: d_{i} \in S\right\}\right| \leq u\right\} .
\end{aligned}
$$

Establishing domain consistency for the among constraint is not difficult. Subtracting from $\ell, u$, and $q$ the number of variables that must take their value in $S$, and subtracting further from $q$ the number of variables that cannot take their value in $S$, we are in one of four cases:

1. $u<0$ or $\ell>q$ : the constraint is inconsistent;

2. $u=0$ : remove values in $S$ from the domain of all remaining variables, making the constraint domain consistent;

3. $\ell=q$ : remove values not in $S$ from the domain of all remaining variables, making the constraint domain consistent;

4. $u>0$ and $\ell<q$ : the constraint is already domain consistent.

The sequence constraint applies the same among constraint on every $q$ consecutive variables:

Definition 3 (Sequence constraint, [3]) Let $X=x_{1}, x_{2}, \ldots, x_{n}$ be an ordered sequence of variables (according to their respective indices) and let $S$ be a set of domain values. Let $1 \leq q \leq n$ and $0 \leq \ell \leq u \leq q$ be constants. Then

$$
\text { sequence }(X, S, q, \ell, u)=\bigwedge_{i=1}^{n-q+1} \operatorname{among}\left(s_{i}, S, \ell, u\right),
$$

where $s_{i}$ represents the subsequence $x_{i}, \ldots, x_{i+q-1}$.

In words, the sequence constraint states that at least $\ell$ and at most $u$ values in $S$ are assigned to every subsequence of $q$ consecutive variables. Note that working on each among constraint separately, and hence locally, is not as powerful as reasoning globally. In particular, as the following example shows, establishing domain consistency on each among of the conjunction does not ensure domain consistency for sequence. 
Example 1 Let $X=x_{1}, x_{2}, x_{3}, x_{4}, x_{5}, x_{6}, x_{7}$ be an ordered sequence of variables with domains $D\left(x_{i}\right)=\{0,1\}$ for $i \in\{3,4,5,7\}, D\left(x_{1}\right)=D\left(x_{2}\right)=\{1\}$, and $D\left(x_{6}\right)=\{0\}$. Consider the constraint sequence $(X,\{1\}, 5,2,3)$, i.e., every sequence of five consecutive variables must account for two or three 1's. Each individual among is domain consistent but it is not the case for sequence: value 0 is unsupported for variable $x_{7}$. ( $x_{7}=0$ forces at least two 1's among $\left\{x_{3}, x_{4}, x_{5}\right\}$, which brings the number of 1's for the leftmost among to at least four.)

Establishing domain consistency for the sequence constraint is not nearly as easy as for among. The algorithms proposed so far in the literature may miss such global reasoning. For instance, the filtering algorithm proposed by Régin and Puget [13] and implemented in Ilog Solver does not filter out 0 from $D\left(x_{7}\right)$ in Example 1.

Remark 1 When $\ell$ equals $u$, domain consistency can in fact be established in linear time. Specifically, if there is a solution, then $x_{i}$ must equal $x_{i+q}$ because of the constraints $a_{i}+a_{i+1}+\cdots+a_{i+q-1}=\ell$ and $a_{i+1}+\cdots+a_{i+q}=\ell$. Hence, if one divides the sequence up into $n / q$ consecutive subsequences of size $q$ each, they must all look identical. Thus, establishing domain consistency now amounts to propagating the "settled" variables (i.e. $x_{i}$ for which $D\left(x_{i}\right) \subseteq S$ or $D\left(x_{i}\right) \cap S=\varnothing$ ) to the first subsequence and then applying the previously described algorithm for among. Two of the filtering algorithms we describe in this article establish domain consistency in the general case, i.e., when $\ell$ does not necessarily equal $u$.

Without loss of generality, we shall consider instances of sequence in which $S=\{1\}$ and the domain of each variable is a subset of $\{0,1\}$. Indeed, using the element $\left(x, t, x^{\prime}\right)$ constraint, which states that $x^{\prime}$ is equal to the value in table $t$ that is indexed by $x$, we can define $t$ to map every value in $S$ to 1 and every other value (i.e., $D(X) \backslash S)$ to 0 , yielding an equivalent instance on new variables. For example, let $D(x)=\{1,2,3,4,5\}$ for some variable $x$ and $S=\{1,2,4\}$. The constraint element $\left(x,[1,1,0,1,0], x^{\prime}\right)$ yields $D\left(x^{\prime}\right)=\{0,1\}$ and $x^{\prime}=1$ if and only if $x \in S$. An among or sequence constraint restricting the number of times $x$ takes values in $S$ can thus be equivalently represented by a constraint restricting the number of times $x^{\prime}$ takes the value 1 .

\section{A Graph-Based Filtering Algorithm}

We first propose a filtering algorithm that considers the individual among constraints of which the sequence constraint is composed. It begins by filtering the among constraints for each sequence of $q$ consecutive variables $s_{i}$, similar to the dynamic programming approach taken by Trick [14] to filter knapsack constraints. It then filters the conjunction of every pair of consecutive sequences $s_{i}$ and $s_{i+1}$. This is presented as SuccessiveLocalGraph (SLG) in Algorithm 1, and discussed below.

\subsection{Filtering Individual among Constraints}

The individual among constraints are filtered with the algorithm FILTERLOCALGRAPH. For each sequence $s_{i}=x_{i}, \ldots, x_{i+q-1}$ of $q$ consecutive variables in $X=$ 
$x_{1}, \ldots, x_{n}$, we build a directed graph $G_{s_{i}}=\left(V_{i}, A_{i}\right)$ as follows. The vertex set and the arc set are defined as

$$
\begin{gathered}
V_{i}=\left\{v_{j, k} \mid j \in\{i-1, \ldots, i+q-1\}, k \in\{0, \ldots, j\}\right\}, \\
A_{i}=\left\{\left(v_{j, k}, v_{j+1, k}\right) \mid j \in\{i-1, \ldots, i+q-2\}, k \in\{0, \ldots, j\}, D\left(x_{j+1}\right) \backslash S \neq \emptyset\right\} \bigcup \\
\left\{\left(v_{j, k}, v_{j+1, k+1}\right) \mid j \in\{i-1, \ldots, i+q-2\}, k \in\{0, \ldots, j\}, D\left(x_{j+1}\right) \cap S \neq \emptyset\right\} .
\end{gathered}
$$

In words, the arc $\left(v_{j, k}, v_{j+1, k+1}\right)$ in the graphs represents variable $x_{j+1}$ taking its value in $S$, while the arc $\left(v_{j, k}, v_{j+1, k}\right)$ represents variable $x_{j+1}$ not taking its value in $S$. The index $k$ in $v_{j, k}$ represents the number of variables in $x_{i}, \ldots, x_{j}$ that take their value in $S$.

For each local graph $G_{s_{i}}$, we define a set of goal vertices as $\left\{v_{i+q-1, k} \mid \ell \leq k \leq u\right\}$. We then have the following immediate result:

Lemma 1 A solution to the among constraint on sequence $s_{i}$ corresponds to a directed path from $v_{i-1,0}$ to a goal vertex in $G_{s_{i}}$, and vice versa.

Next we apply Lemma 1 to make individual among constraints domain consistent. For the among constraint on sequence $s_{i}$, we remove all arcs that are not on any path from $v_{i-1,0}$ to a goal vertex in $G_{s_{i}}$. This can be done in linear time (in the size of the graph, $\Theta\left(q^{2}\right)$ ) by breadth-first search starting from the goal vertices. If the filtered graph contains no arc $\left(v_{j, k}, v_{j+1, k}\right)$ for all $k$, we remove $S$ from $D\left(x_{j+1}\right)$. Similarly, we remove $D(X) \backslash S$ from $D\left(x_{j+1}\right)$ if it contains no $\operatorname{arc}\left(v_{j, k}, v_{j+1, k+1}\right)$ for all $k$.

Example 2 Let $X=x_{1}, x_{2}, x_{3}, x_{4}, x_{5}, x_{6}$ be an ordered sequence of variables with domains $D\left(x_{i}\right)=\{0,1\}$ for $i \in\{1,2,3,4,6\}$ and $D\left(x_{5}\right)=\{1\}$. Let $S=\{1\}$. Consider the constraint sequence $(X, S, 4,2,2)$. The filtered local graphs of this constraint are depicted in Figure 1.
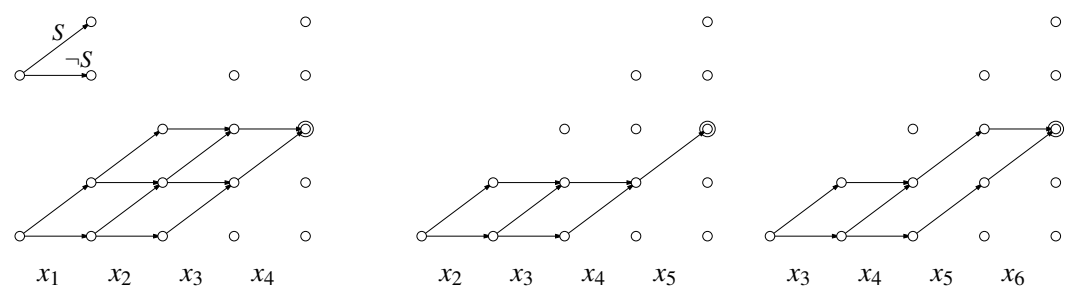

Fig. 1 Filtered Local Graphs of Example 2 


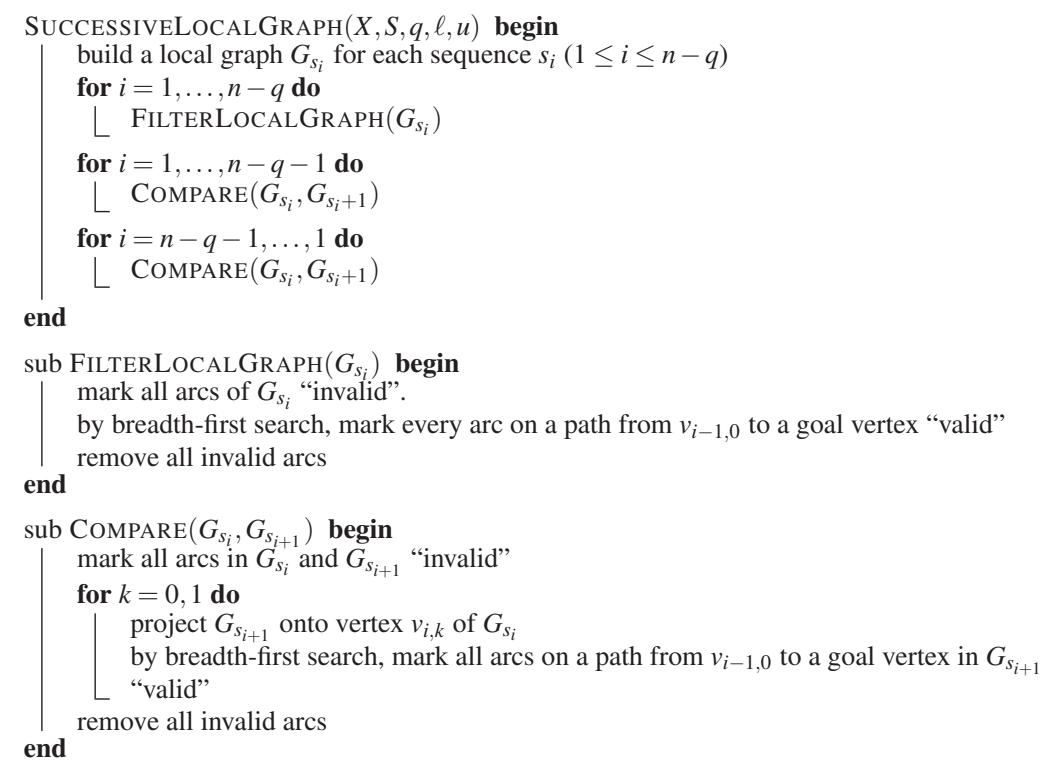

Algorithm 1: A graph-based filtering algorithm for the sequence constraint

\subsection{Filtering a Sequence of among Constraints}

We next filter the conjunction of two "consecutive" among constraints. Our algorithm has a "forward" phase and a "backward" phase. In the forward phase, we compare the among on $s_{i}$ with the among on $s_{i+1}$ for increasing $i$, using the algorithm COMPARE (see Algorithm 1). This is done by projecting $G_{s_{i+1}}$ onto $G_{s_{i}}$ such that corresponding variables overlap. Doing so, the projection keeps only arcs that appear in both original local graphs. We can either project vertex $v_{i+1,0}$ of $G_{s_{i+1}}$ onto vertex $v_{i+1,0}$ of $G_{s_{i}}$, or onto vertex $v_{i+1,1}$ of $G_{s_{i}}$. We consider both projections separately, and label all arcs "valid" if they belong to a path from vertex $v_{i, 0}$ to goal vertex in $G_{s_{i+1}}$ in at least one of the composite graphs. All other arcs are labeled "invalid", and are removed from both the original graphs, $G_{s_{i}}$ and $G_{s_{i+1}}$. In the backward phase, we compare the among on $s_{i}$ with the among on $s_{i+1}$ for decreasing $i$, similar to the forward phase.

\subsection{Analysis}

The time complexity of SUCCESSIVELOCALGRAPH is polynomial since the local graphs are all of size $\mathrm{O}(q \cdot u)$. Hence FilterLocalGraPH runs in $\mathrm{O}(q \cdot u)$ time, which is called $n-q$ times. The algorithm COMPARE similarly runs for $\mathrm{O}(q \cdot u)$ steps and is called $2(n-q)$ times. Thus, the filtering algorithm runs in $\mathrm{O}((n-q) \cdot q \cdot u)$ time. As $u \leq q$, it follows that the algorithm runs in $\mathrm{O}\left(n q^{2}\right)$ time.

As mentioned earlier, SuccessiveLocalGraph does not establish domain consistency for the sequence constraint. We illustrate this with the following example. 
Example 3 Let $X=x_{1}, x_{2}, \ldots, x_{10}$ be an ordered sequence of variables with domains $D\left(x_{i}\right)=\{0,1\}$ for $i \in\{3,4,5,6,7,8\}$ and $D\left(x_{i}\right)=\{0\}$ for $i \in\{1,2,9,10\}$. Let $S=$ $\{1\}$. Consider the constraint sequence $(X, S, 5,2,3)$, i.e., every sequence of 5 consecutive variables must take between 2 and 3 values in $S$. The first among constraint imposes that at least two variables out of $\left\{x_{3}, x_{4}, x_{5}\right\}$ must be 1 . Hence, at most one variable out of $\left\{x_{6}, x_{7}\right\}$ can be 1 , by the third among. This implies that $x_{8}$ must be 1 (from the last among). Similarly, we can deduce that $x_{3}$ must be 1 . This is, however, not deduced by our algorithm, as can be readily verified.

The problem occurs in the COMPARE method, when we merge the valid arcs coming from the different projections. Up until that point there is a direct equivalence between a path in a local graph and a support for the constraint. However the union of the two projections breaks this equivalence and thus prevents this algorithm from establishing domain consistency.

\section{Reaching Domain Consistency Through regular}

The regular constraint [10], defining the set of allowed tuples for a sequence of variables as the language recognized by a given automaton, admits an incremental filtering algorithm establishing domain consistency. In this section, we give an automaton recognizing the tuples of the sequence constraint whose number of states is potentially exponential in $q$. Through that automaton, we can express sequence as a regular constraint, thereby obtaining domain consistency.

The idea is to record in a state the last $q$ values encountered, keeping only the states representing valid numbers of 1's for a sequence of $q$ consecutive variables and adding the appropriate transitions between those states. Let $Q_{k}^{q}$ denote the set of strings of length $q$ featuring exactly $k$ 1's and $q-k 0$ 's; there are $\left(\begin{array}{l}q \\ k\end{array}\right)$ such strings. Given the constraint sequence $(X,\{1\}, q, \ell, u)$, we create states for each of the strings in $\bigcup_{k=\ell}^{u} Q_{k}^{q}$. By a slight abuse of notation, we will refer to a state using the string it represents. Consider a state $d_{1} d_{2} \ldots d_{q}$ in $Q_{k}^{q}, \ell \leq k \leq u$. We add a transition on 0 to state $d_{2} d_{3} \ldots d_{q} 0$ if and only if $d_{1}=0 \vee\left(d_{1}=1 \wedge k>\ell\right)$. We add a transition on 1 to state $d_{2} d_{3} \ldots d_{q} 1$ if and only if $d_{1}=1 \vee\left(d_{1}=0 \wedge k<u\right)$.

We must add some other states to encode the first $q-1$ values of the sequence: one for the initial state, two to account for the possible first value, four for the first two values, and so forth. There are at most $2^{q}-1$ of those states, considering that some should be excluded because the number of 1 's does not fall within $[\ell, u]$. More precisely, we will have states

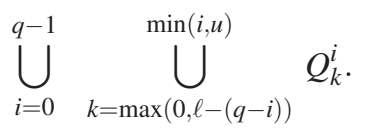

We define transitions from a state $d_{1} \ldots d_{i}$ in $Q_{k}^{i}$ to state $d_{1} \ldots d_{i} 0$ in $Q_{k}^{i+1}$ on value 0 and to state $d_{1} \ldots d_{i} 1$ in $Q_{k+1}^{i+1}$ on value 1 , provided such states are part of the automaton. Every state in the automaton is considered a final (accepting) state. Figure 2 illustrates the automaton that would be built for the constraint sequence $(X,\{1\}, 4,1,2)$. 

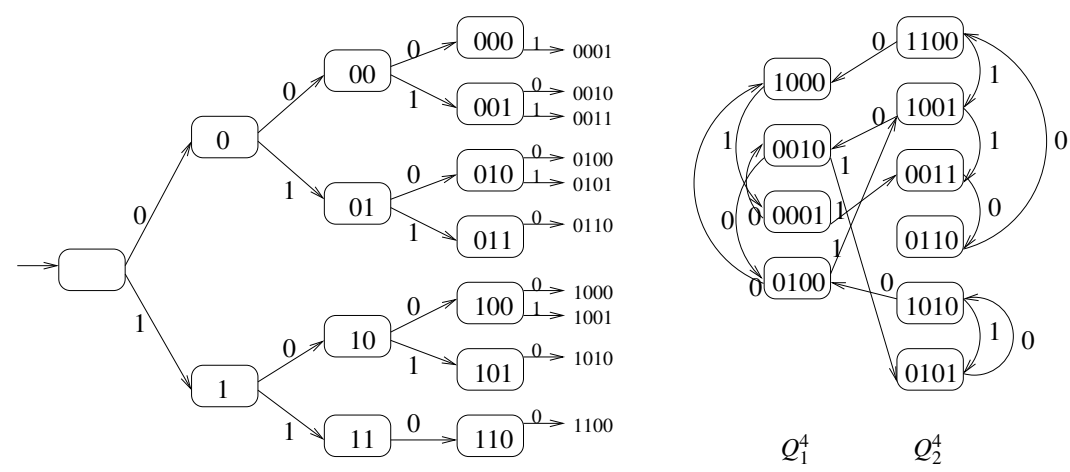

$Q_{0}^{0} \quad Q_{0}^{1} \cup Q_{1}^{1} \quad Q_{0}^{2} \cup Q_{1}^{2} \cup Q_{2}^{2} \quad Q_{0}^{3} \cup Q_{1}^{3} \cup Q_{2}^{3}$

Fig. 2 Automaton for sequence $(X,\{1\}, 4,1,2)$

The filtering algorithm for regular guarantees domain consistency provided that the automaton recognizes precisely the solutions of the constraint. By construction, the states $Q_{\star}^{q}$ of the automaton represent all the valid configurations of $q$ consecutive values and the transitions between them imitate a shift to the right over the sequence of values. In addition, the states $Q_{\star}^{i}, 0 \leq i<q$ are linked so that the first $q$ values reach a state that encodes them. All states are accepting states so the sequence of $n$ values is accepted if and only if the automaton completes the processing. Such a completion corresponds to a successful scan of every subsequence of length $q$, precisely our solutions.

The resulting algorithm runs in time linear in the size of the underlying graph, which has $\mathrm{O}\left(n 2^{q}\right)$ vertices and arcs in the worst case. Nevertheless, in many practical problems $q$ is much smaller than $n$. Note also that subsequent calls to the algorithm run in time proportional to the number of updates in the graph and not to the size of the whole graph.

\section{Reaching Domain Consistency in Polynomial Time}

The filtering algorithms we considered thus far apply to sequence constraints with fixed among constraints for the same $q, \ell$, and $u$. In this section we present a polynomial-time algorithm that achieves domain consistency in a more generalized setting, where we have $m$ arbitrary among constraints over sequences of consecutive variables in $X$. These $m$ constraints may have different $\ell$ and $u$ values, be of different length, and overlap in an arbitrary fashion. However, they must be defined using the same set of values $S$. A conjunction of $k$ sequence constraints over the same ordered set of variables, for instance, can be expressed as a single generalized sequence constraint. We define the generalized sequence constraint, gen-sequence, formally as follows:

Definition 4 (Generalized sequence constraint) Let $X=x_{1}, \ldots, x_{n}$ be an ordered sequence of variables (according to their respective indices) and $S$ be a set of domain 
values. For $1 \leq j \leq m$, let $s_{j}$ be a sequence of consecutive variables in $X,\left|s_{j}\right|$ denote the length of $s_{j}$, and integers $\ell_{j}$ and $u_{j}$ be such that $0 \leq \ell_{j} \leq u_{j} \leq\left|s_{j}\right|$. Let $\Sigma=$ $\left\{s_{1}, \ldots, s_{m}\right\}, \mathrm{L}=\left\{\ell_{1}, \ldots, \ell_{m}\right\}$, and $\mathrm{U}=\left\{u_{1}, \ldots, u_{m}\right\}$. Then

$$
\text { gen-sequence }(X, S, \Sigma, \mathrm{L}, \mathrm{U})=\bigwedge_{j=1}^{m} \operatorname{among}\left(s_{j}, S, \ell_{j}, u_{j}\right) \text {. }
$$

For simplicity, we will identify each $s_{j} \in \Sigma$ with the corresponding among constraint on $s_{j}$. As before, we will assume without loss of generality that $D\left(x_{i}\right) \subseteq\{0,1\}$ and $S=\{1\}$. The basic structure of the filtering algorithm for the gen-sequence constraint is presented as Algorithm 2. The main loop, COMPLETEFILTERINGGS, is based on the standard shaving process, which works as follows. If a variable-value pair is yet unsupported, we temporarily make the corresponding variable assignment and check its consistency via procedure CHECKCONSISTENCY. If this assignment makes the constraint inconsistent, we remove the value from the domain of the variable under consideration; otherwise we (implicitly) mark this variable-value pair as supported.

Procedure CHeckConsistency is the heart of the algorithm. It finds one solution to the gen-sequence constraint, or proves that none exists. It uses a single array $y$ of length $n+1$, such that $y[0]=0$ and $y[i]$ represents the number of 1's among $x_{1}, \ldots, x_{i}$. The invariant for $y$ maintained throughout is that $y[i+1]-y[i]$ is either 0 or 1. Initially, we start with the lowest possible array, in which $y$ is filled according to the lower bounds of the variables in $X$.

For clarity, let $L_{j}$ and $R_{j}$ denote the left and right end-points, respectively, of the among constraint $s_{j} \in \Sigma$; note that $R_{j}=L_{j}+\left|s_{j}\right|-1$. As an example, for the usual sequence constraint with among constraints of size $q, L_{j}$ would be $j$ and $R_{j}$ would be $j+q-1$. The value of $s_{j}$ is computed using the array $y$ : value $\left(s_{j}\right)=y\left[R_{j}\right]-y\left[L_{j}-\right.$ 1]. In other words, value $\left(s_{j}\right)$ counts exactly the number of 1 's in the sequence $s_{j}$. Hence, the constraint $s_{j}$ is satisfied if and only if $\ell_{j} \leq \operatorname{value}\left(s_{j}\right) \leq u_{j}$. In order to find a solution, we consider all among constraints $s_{j} \in \Sigma$. Whenever a constraint $s_{j}$ is violated, we make it consistent by "pushing up" either $y\left[R_{j}\right]$ or $y\left[L_{j}-1\right]$ :

if value $\left(s_{j}\right)<\ell_{j}$, then push up $y\left[R_{j}\right]$ with value $\ell_{j}-\operatorname{value}\left(s_{j}\right)$, if value $\left(s_{j}\right)>u_{j}$, then push up $y\left[L_{j}-1\right]$ with value value $\left(s_{j}\right)-u_{j}$.

Such a "push up" may result in the invariant for $y$ being violated. We therefore repair $y$ in a minimal fashion to restore its invariant as follows. Let $y[i d x]$ be the entry that has been pushed up. We first push up its neighbors on the left side (from idx downward), starting with $i d x-1$. In case $x_{i d x-1}$ is fixed to 0 , we push up $y[i d x-1]$ to the same level $y[i d x]$. Otherwise, we push it up to $y[i d x]-1$. This continues until the difference between all neighbors to the left of $i d x$ is at most 1 and respects the values of the fixed variables. Whenever $y[i]>i$ for some $i$ during this process, this indicates that we need more 1's than there are variables up to $i$, and we therefore report an immediate failure. Repairing the array on the right side of $i d x$ is done in a similar manner. Here, in case $x_{i d x+1}$ is fixed to 1 , we push up $y[i d x+1]$ to $y[i d x]+1$. Otherwise, we push it up to the same level $y[i d x]$. The process continues to the right as far as necessary. 


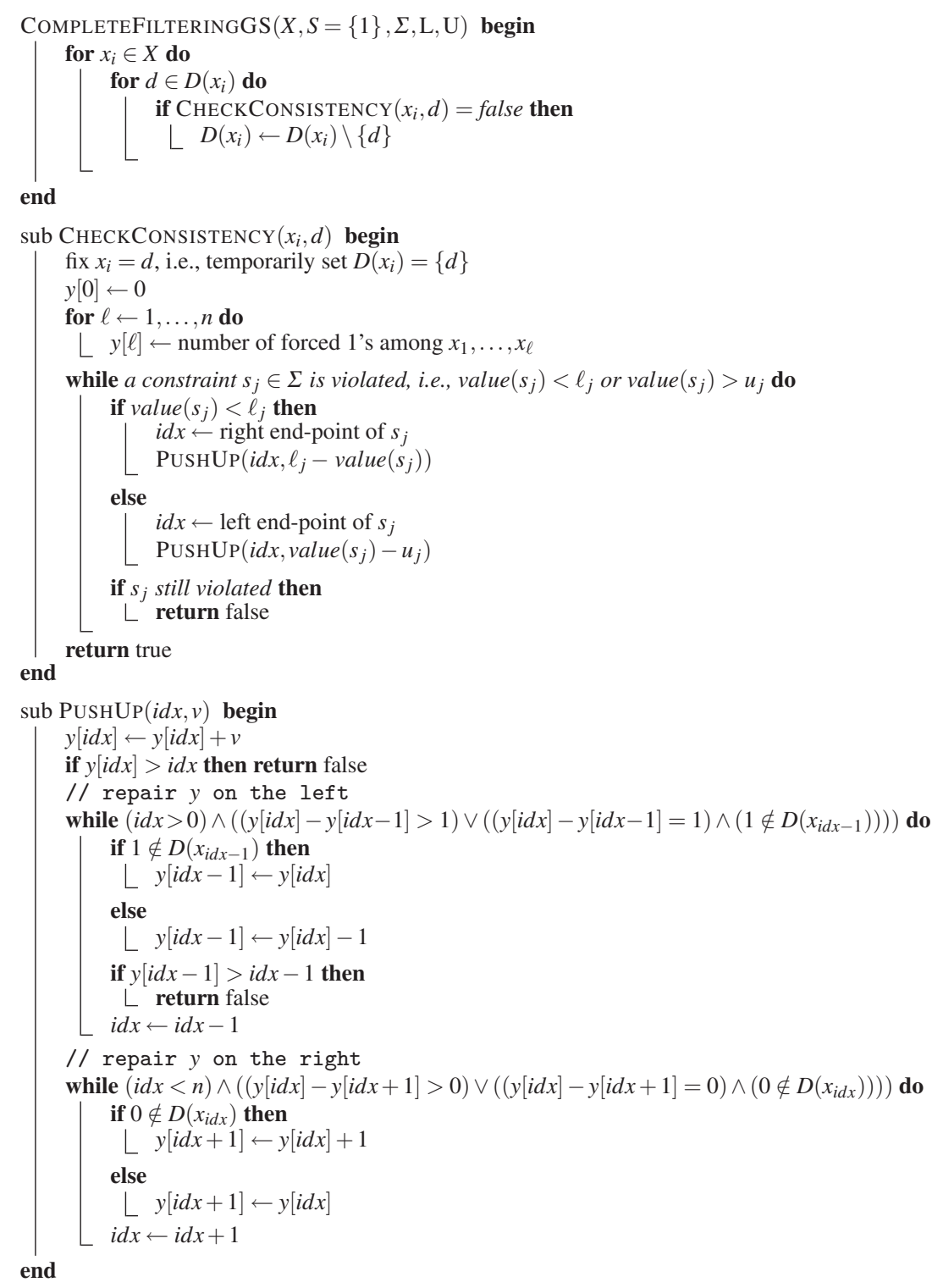

Algorithm 2: Complete filtering algorithm for the gen-sequence constraint

Example 4 Consider the constraint sequence $(X, S, 3,2,2)$ with $X=$ $\left\{x_{1}, x_{2}, x_{3}, x_{4}, x_{5}, x_{6}\right\}, D\left(x_{i}\right)=\{0,1\}$ for $i \in\{1,2,3,4,6\}, D\left(x_{5}\right)=\{1\}$, and $S=\{1\}$. The four among constraints are over $s_{1}=\left\{x_{1}, x_{2}, x_{3}\right\}, s_{2}=\left\{x_{2}, x_{3}, x_{4}\right\}$, $s_{3}=\left\{x_{3}, x_{4}, x_{5}\right\}$, and $s_{4}=\left\{x_{4}, x_{5}, x_{6}\right\}$. We apply CHECKCONSISTENCY to find the "minimum" solution to this constraint. (We defer a formal discussion of the minimum solution to next subsection.) The various steps are depicted in Figure 3. 

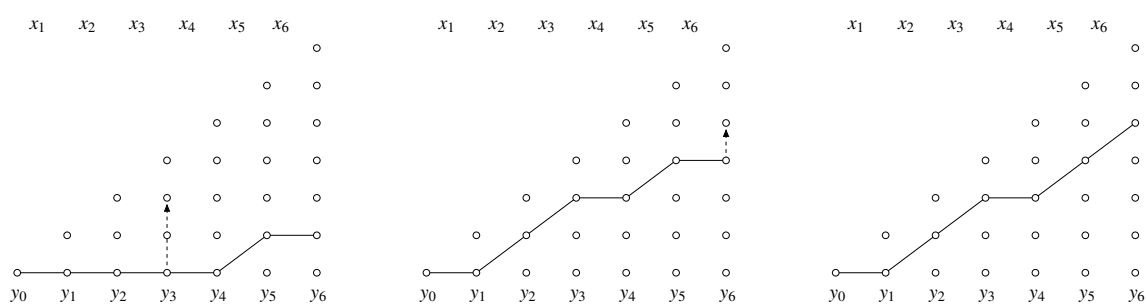

Fig. 3 Finding a minimum solution to Example 4

We start with $y=[0,0,0,0,0,1,1]$ because $x_{5}$ is forced to be 1 , and then evaluate the different among constraints to check whether any of them is violated. We first consider $s_{1}$, which is violated: value $\left(s_{1}\right)=y[3]-y[0]=0-0=0$, while it should be at least 2 . Hence, we push up $y[3]$ by 2 units, and obtain $y=[0,0,1,2,2,3,3]$ after repairs. Note that we push up $y[5]$ to 3 because $x_{5}$ is fixed to 1 . Next we consider $s_{2}$ with value $y[4]-y[1]=2$, which is not violated. We continue with $s_{3}$ with value $y[5]-y[2]=2$, which is not violated. Then we consider $s_{4}$ with value $y[6]-y[3]=1$, which is violated as it should be at least 2 . Hence, we push up $y[6]$ by 1 , and obtain $y=[0,0,1,2,2,3,4]$. As there are no more violated among constraints, we conclude consistency, with minimum solution $x_{1}=0, x_{2}=1, x_{3}=1, x_{4}=0, x_{5}=1, x_{6}=1$.

The basic procedure in Algorithm 2 can be optimized in several ways; our implementation includes these optimizations. The main loop of COMPLETEFILTERINGGS is improved by maintaining a support for all domain values. Specifically, one call to CHECKCONSISTENCY (with a positive response) yields a support for $n$ domain values. This immediately reduces the number of calls to CHECKCONSISTENCY by half, while in practice the cumulative reduction is even more. A second improvement is achieved by starting out COMPLETEFILTERINGGS with the computation of the "minimum" and the "maximum" solutions to gen-sequence, in a manner very similar to the computation in CHECKCONSISTENCY but without restricting the value of any variable. This defines bounds $y_{\min }$ and $y_{\max }$ within which $y$ must lie for all subsequent consistency checks (details are presented in the following section). As we will shortly see, this is further generalized to maintaining one minimum and one maximum solution for each variable-value pair, yielding an improvement in the cumulative time complexity of the algorithm from the root of the search tree to any leaf.

\subsection{Analysis}

A solution to a gen-sequence constraint can be thought of as the corresponding binary sequence given by the $x$ array or, equivalently, as the cumulative $y$ array. This $y$ array representation has a useful property which we use for analyzing the correctness and the complexity of the algorithm. Let $y$ and $y^{\prime}$ be two solutions. Define array $y \oplus y^{\prime}$ to be the smaller of $y$ and $y^{\prime}$ at each point, i.e., $\left(y \oplus y^{\prime}\right)[i]=\min \left(y[i], y^{\prime}[i]\right)$.

Lemma 2 If $y, y^{\prime}$ are solutions to a gen-sequence constraint, then so is $y \oplus y^{\prime}$. 
Proof Suppose for the sake of contradiction that $y^{*}=y \oplus y^{\prime}$ violates an among constraint $s$ of the gen-sequence constraint. Let $L$ and $R$ denote the left and right endpoints of $s$, respectively. Suppose $y^{*}$ violates the $\ell$ constraint, i.e., $y^{*}[R]-y^{*}[L-1]<$ $\ell(s)$. Since $y$ and $y^{\prime}$ satisfy $s$, it must be that $y^{*}$ agrees with $y$ on one end-point of $s$ and with $y^{\prime}$ on the other. W.l.o.g., assume $y^{*}[L-1]=y^{\prime}[L-1]$ and $y^{*}[R]=y[R]$. By the definition of $y^{*}$, it must be that $y[L-1] \geq y^{\prime}[L-1]$, so that $y[R]-y[L-1] \leq$ $y[R]-y^{\prime}[L-1]=y^{*}[R]-y^{*}[L-1]<\ell(s)$. In other words, $y$ itself violates $s$, a contradiction. A similar reasoning works when $y^{*}$ violates the $u$ constraint of $s$.

As a consequence of this property, we can unambiguously define an absolute minimum solution for gen-sequence as the one whose $y$ value is the point-wise lowest over all solutions. Denote this solution by $y_{\min }$; we have that for all solutions $y$ and for all $i, y_{\min }[i] \leq y[i]$. Similarly, define the absolute maximum solution, $y_{\max }$. For clarity, we will only focus on the minimum solution, which suffices for the proofs that follow; our implementation uses both the minimum and the maximum solutions to reduce the running time in practice.

Lemma 3 The procedure CHECKCONSISTENCY constructs the minimum solution to the sequence constraint and the gen-sequence constraint, or proves that none exists, in time $\mathrm{O}\left(n^{2}\right)$ and $\mathrm{O}\left(n^{3}\right)$, respectively.

Proof CHeckConsistency reports success only when no among constraint in gen-sequence is violated by the current $y$ values maintained by it, i.e., $y$ is a solution. Hence, if there is no solution, this fact is detected. We will argue that if there is a solution, CHECKCONSISTENCY reports success and its current $y$ array exactly equals $y_{\min }$.

We first show by induction that $y$ never goes above $y_{\min }$ at any point, i.e., $y[i] \leq$ $y_{\min }[i], 0 \leq i \leq n$ throughout the procedure. For the base case, $y[i]$ is clearly initialized to a value not exceeding $y_{\min }[i]$, and the claim holds trivially. Assume inductively that the claim holds after processing $t \geq 0$ among constraint violations. Let $s$ be the $t+1^{s t}$ violated constraint processed. We will show that the claim still holds after processing $s$.

Let $L$ and $R$ denote the left and right end-points of $s$, respectively. First consider the case that the $\ell$ constraint was violated, i.e., $y[R]-y[L-1]<\ell(s)$, and index $R$ was pushed up so that the new value of $y[R]$, denoted $\hat{y}[R]$, became $y[L-1]+\ell(s)$. Since this was the first time a $y$ value exceeded $y_{\min }$, we have $y[L-1] \leq y_{\min }[L-1]$, so that $\hat{y}[R]=y[L-1]+\ell(s) \leq y_{\min }[L-1]+\ell(s) \leq y_{\min }[R]$. Therefore, $\hat{y}[R]$ itself does not exceed $y_{\min }[R]$. It may, in principle, still be the case that the resulting repair on the left or the right causes a $y_{\min }$ violation. However, the repair operations only lift up $y$ values barely enough to be consistent with the possible domain values of the relevant variables. In particular, repair on the right "flattens out" $y$ values to equal $\hat{y}[L-1]$ (forced 1's being exceptions) as far as necessary to "hit" the solution again. It follows that since $\hat{y}[R] \leq y_{\min }[R]$, all repaired $y$ values must also not go above $y_{\min }$. A similar argument works when instead the $u$ constraint is violated. This finishes the inductive step.

This shows that by performing repeated PUSHUP operations, one can never accidentally "go past" the solution $y_{\min }$ at any point. Further, since each PUSHUP in- 
creases $y$ in at least one place, repeated calls to it will eventually "hit" $y_{\min }$ as a solution.

For the time complexity of CHeCKConsistency, note that $y[i] \leq i$. Since we monotonically increase the $y$ values, we can do so at most $\sum_{i=1}^{n} i=\mathrm{O}\left(n^{2}\right)$ times. The cost of each PUSHUP operation can be charged to the $y$ values it changes because the while loops in it terminate as soon as they find a $y$ value that need not be changed.

Finally, we discuss the detection of the violated among constraints during CHECKCONSISTENCY. For this, we maintain a stack of indices, corresponding to (possibly) violated constraints. When processing a constraint, we remove it from the stack, and potentially push up some indices to make it consistent. Whenever we push up $y_{i}$, we insert all violated among constraints that have $i$ as a left or right endpoint. In the case of the normal sequence constraint, we check in constant time whether $s_{i}$ or $s_{i+1-q}$ should be added to the stack. This yields the desired overall time complexity of $\mathrm{O}\left(n^{2}\right)$. Notice that the stack is of size $\mathrm{O}\left(n^{2}\right)$, because we only insert pushedup indices, of which there are at most $\mathrm{O}\left(n^{2}\right)$ during the process. In the case of the gen-sequence constraint, we maintain additionally two vectors indicating for each index $i$ the among constraints that start, respectively end, at $i$. Notice that at most $n$ constraints can start or end at an index $i$. Using this representation, the detection of violated among constraints for pushed up index $i$ takes at most $\mathrm{O}(n)$ time, which results in a net worst-case time complexity of $\mathrm{O}\left(n^{3}\right)$ for the gen-sequence constraint.

Theorem 1 Algorithm COMPLETEFILTERINGGS establishes domain consistency on the gen-sequence constraint. Further, it can be implemented such that along every path from the root to a leaf of the search tree, it takes time $\mathrm{O}\left(n^{3}\right)$ for the sequence constraint and $\mathrm{O}\left(n^{4}\right)$ for the gen-sequence constraint.

Proof Lemma 3 and the simple loop structure of COMPLETEFILTERINGGS together imply that we obtain domain consistency (or prove inconsistency) each time the algorithm is invoked. To obtain the desired time complexity from the root to a leaf of the search tree, we maintain for every variable-value pair a minimum $y$ solution. There are $\mathrm{O}(n)$ such minimum solutions, each of size $n$. When checking consistency of such a pair, we start from the corresponding minimum $y$ solution and update it if we find a new one at this point in the search tree. As we proceed from the root to a leaf in the search tree, these minimum solutions can only (point-wise) increase. Moreover, the number of push-ups is at most $\mathrm{O}\left(n^{2}\right)$ for each variable-value pair from the root to a leaf, following the same reasoning as in the proof of Lemma 3. It follows that for all $n$ variables, we obtain a time complexity of $\mathrm{O}\left(n^{3}\right)$ for the sequence constraint, and $\mathrm{O}\left(n^{4}\right)$ for the gen-sequence constraint, along every path from the root to a leaf in the search tree.

\section{Experimental Results}

To evaluate the different filtering algorithms presented, we used three sets of benchmark problems. The first is a very simple model, constructed with only one sequence constraint, allowing us to isolate and evaluate the performance of each method separately. We then report the results of a series of experiments on the well-known car 
Table 1 Comparison on instances with $n=100, d=10$

\begin{tabular}{|c|c|c|c|c|c|c|c|c|c|c|c|}
\hline \multirow{2}{*}{\multicolumn{2}{|c|}{$q \Delta$}} & \multicolumn{2}{|c|}{ IB } & \multicolumn{2}{|c|}{ IE } & \multicolumn{2}{|c|}{ SLG } & \multicolumn{2}{|c|}{ REG } & \multicolumn{2}{|c|}{ GS } \\
\hline & & BT & CPU & BT & CPU & BT & CPU & BT & CPU & BT & CPU \\
\hline 5 & $\overline{1}$ & - & - & $33,976.9$ & 18.210 & 0.2 & 0.069 & 0 & 0.009 & 0 & 0.014 \\
\hline 6 & 2 & 361,770 & 54.004 & $19,058.3$ & 6.390 & 0 & 0.078 & 0 & 0.018 & 0 & 0.013 \\
\hline 7 & 1 & 380,775 & 54.702 & $113,166.0$ & 48.052 & 0 & 0.101 & 0 & 0.020 & 0 & 0.012 \\
\hline 7 & 2 & 264,905 & 54.423 & $7,031.0$ & 4.097 & 0 & 0.129 & 0 & 0.039 & 0 & 0.016 \\
\hline 7 & 3 & 286,602 & 48.012 & 0 & 0.543 & 0 & 0.129 & 0 & 0.033 & 0 & 0.015 \\
\hline 9 & 1 & - & - & $60,780.5$ & 42.128 & 0.1 & 0.163 & 0 & 0.059 & 0 & 0.010 \\
\hline 9 & 3 & 195,391 & 43.024 & 0 & 0.652 & 0 & 0.225 & 0 & 0.187 & 0 & 0.016 \\
\hline
\end{tabular}

sequencing problem. Finally, we consider a combination of sequence constraints of varied lengths, to evaluate the gen-sequence constraint.

In the following, Successive Local Graph (SLG), regular-based implementation (REG), and Generalized Sequence (GS) are compared with the sequence constraint implementation provided in the Ilog Solver library in both basic (IB) and extended (IE) propagation modes. Experiments were run with Ilog Solver 6.2 on a dual processor Intel Xeon HT 2.8Ghz machine with 3GB RAM.

\subsection{A Single Sequence Constraint}

To evaluate the filtering both in terms of domain reduction and efficiency, we build a very simple model consisting of only one sequence constraint. The instances are generated in the following manner. All instances contain $n$ variables of domain size $d$ and the set $S$ is composed of the first $d / 2$ elements. We generate a family of instances by varying the sequence size $q$ and the difference between $\ell$ and $u, \Delta=u-\ell$. For each family we try to generate 10 challenging instances by randomly filling the domain of each variable and by enumerating all possible values of $\ell$. These instances are then solved using a random choice for both variable and value selection, keeping only the ones that are solved with more than 10 backtracks by method IB. All runs were stopped after one minute of computation. The runtimes in seconds (CPU) and the number of backtracks (BT) are reported as the average over various instances with the same parameters.

Table 1 reports on instances with a fixed number of variables $(n=100)$ and varying $q$ and $\Delta$. Table 2 reports on instances with a fixed $\Delta(=1)$ and a growing number $n$ of variables.

These results demonstrate that the new filtering algorithms are very efficient. Both REG and GS require no backtracks because they achieve domain consistency. The average number of backtracks for SLG is also typically very low. As predicted by its time complexity, GS is very stable for fixed $n$ in the first table but becomes more time consuming as $n$ grows in the second table. The performance of SLG and REG decreases as $q$ grows but REG remains competitive throughout these tests. We expect that the latter would suffer with still larger values of $q$ and $\Delta$ but it proved difficult to generate challenging instances in that range- they tended to be loose enough to be easy for every algorithm. 
Table 2 Comparison on instances with $\Delta=1, d=10$

\begin{tabular}{|c|c|c|c|c|c|c|c|c|c|c|c|}
\hline \multirow[b]{2}{*}{$q$} & \multirow{2}{*}{$\Delta$} & \multicolumn{2}{|c|}{ IB } & \multicolumn{2}{|c|}{ IE } & \multicolumn{2}{|c|}{ SLG } & \multicolumn{2}{|c|}{ REG } & \multicolumn{2}{|c|}{ GS } \\
\hline & & BT & CPU & BT & CPU & BT & CPU & BT & CPU & BT & CPU \\
\hline 5 & 50 & 459,154 & 18.002 & 22,812 & 18.019 & 0.4 & 0.007 & 0 & 0.001 & 0 & 0.001 \\
\hline 5 & 100 & 192,437 & 12.008 & 11,823 & 12.189 & 1.0 & 0.041 & 0 & 0.005 & 0 & 0.005 \\
\hline 5 & 500 & 48,480 & 12.249 & 793 & 41.578 & 0.7 & 1.105 & 0 & 0.023 & 0 & 0.466 \\
\hline 5 & 1000 & 942 & 1.111 & 2.3 & 160.000 & 1.1 & 5.736 & 0 & 0.062 & 0 & 4.374 \\
\hline 7 & 50 & 210,107 & 12.021 & 67,723 & 12.309 & 0.2 & 0.015 & 0 & 0.006 & 0 & 0.001 \\
\hline 7 & 100 & 221,378 & 18.030 & 44,963 & 19.093 & 0.4 & 0.059 & 0 & 0.010 & 0 & 0.005 \\
\hline 7 & 500 & 80,179 & 21.134 & 624 & 48.643 & 2.8 & 2.115 & 0 & 0.082 & 0 & 0.499 \\
\hline 7 & 1000 & 30,428 & 28.270 & 46 & 138.662 & 588.5 & 14.336 & 0 & 0.167 & 0 & 3.323 \\
\hline 9 & 50 & 18,113 & 1.145 & 18,113 & 8.214 & 0.9 & 0.032 & 0 & 0.035 & 0 & 0.001 \\
\hline 9 & 100 & 3,167 & 0.306 & 2,040 & 10.952 & 1.6 & 0.174 & 0 & 0.087 & 0 & 0.007 \\
\hline 9 & 500 & 48,943 & 18.447 & 863 & 65.769 & 2.2 & 4.311 & 0 & 0.500 & 0 & 0.485 \\
\hline 9 & 1000 & 16,579 & 19.819 & 19 & 168.624 & 21.9 & 16.425 & 0 & 0.843 & 0 & 3.344 \\
\hline
\end{tabular}

\subsection{Car Sequencing}

In order to evaluate our algorithms in a more realistic setting, we turned to the car sequencing problem (see prob001 of CSPLib [8] for a detailed description). We ran experiments using the first set of 78 instances on CSPLib. Table 3 compares and contrasts the effectiveness of the following combinations of among constraints. IE, as before, is the extended IloSequence constraint available in the ILOG Solver library. This constraint also allows one to specify individual cardinalities for values in the set $S$, and is thus richer than our basic version of sequence. REG, again as before, is the sequence constraint encoded as a regular constraint. cREG extends REG by including a cost variable [6] that restricts the total number of cars with a particular option. This is more expressive than the normal sequence constraint, but not as rich as the IloSequence constraint IE. Finally, GSa is the gen-sequence constraint that includes the normal sequence constraint along with an additional among constraint restricting the total number of cars with a particular option, similar to cREG and again not as rich as IE.

In addition to these constraints, we also consider the combinations IE+REG, IE+cREG, and IE+GSa, where REG, cREG, and GSa, respectively, are added to the IloSequence constraint as redundant constraints. We note that all versions were run with two different search heuristics: the specialized ordering proposed by Régin and Puget [13] for the car sequencing problems, and the min-domain ordering. In the table, we report the best time (in seconds) for each version. ${ }^{1}$ The time out used for these experiments was one hour.

Table 3 indicates that no single filtering method clearly dominates the others on this relatively complex problem domain. On the positive side, it also shows that our proposed algorithms can be very effective here, either applied solely or as a redundant constraint in conjunction with IloSequence. Specifically, many of the instances can be solved much faster with one of our algorithms than with IloSequence. For example, on all instances that are solved by both GSa and IE, the GSa algorithm achieves a

1 Details of these experiments are available from the authors upon request. 
Table 3 Runtime comparison on car sequencing problems

\begin{tabular}{|c|c|c|c|c|c|c|c|}
\hline instance & IE & REG & cREG & GSa & IE+REG & IE+cREG & IE+GSa \\
\hline carseq01 & 0.39 & 0.04 & 0.06 & 0.03 & 0.41 & 0.49 & 0.42 \\
\hline carseq02 & 83.83 & - & 19.92 & 19.63 & 95.99 & 19.92 & 18.57 \\
\hline carseq03 & 0.60 & - & 0.04 & 0.65 & 0.76 & 0.74 & 0.63 \\
\hline carseq07 & 112.97 & - & 18.24 & 27.70 & 138.19 & 114.98 & 93.38 \\
\hline carseq08 & 0.34 & 0.07 & 0.08 & 0.03 & 0.36 & 0.41 & 0.32 \\
\hline carseq09 & 18.74 & 2253.14 & 21.82 & 16.92 & 22.80 & 20.71 & 18.50 \\
\hline carseq10 & - & - & 1361.94 & 912.00 & - & 4.06 & 3.64 \\
\hline carseq11 & 1.31 & - & 0.46 & 0.20 & 1.32 & 1.66 & 1.67 \\
\hline carseq12 & 24.36 & - & - & - & 23.93 & 38.75 & 48.80 \\
\hline carseq13 & 1.37 & - & 0.35 & 0.18 & 1.40 & 1.76 & 1.53 \\
\hline carseq14 & 2.93 & - & - & - & 2.92 & 3.16 & 3.11 \\
\hline carseq15 & 3.13 & 0.07 & 0.41 & 0.23 & 3.47 & 3.45 & 3.70 \\
\hline carseq16 & 87.09 & - & - & - & 105.28 & 3.50 & 2.84 \\
\hline carseq17 & 2.83 & 0.07 & 0.43 & 0.32 & 3.13 & 3.44 & 3.46 \\
\hline carseq19 & 一 & - & 2.54 & 1.35 & - & - & - \\
\hline carseq20 & - & - & 0.43 & 0.18 & - & 9.58 & 8.51 \\
\hline carseq 21 & 1.54 & - & - & - & 1.56 & 1.92 & 1.69 \\
\hline carseq22 & 451.83 & - & 0.47 & 0.18 & 516.35 & 899.38 & 914.96 \\
\hline carseq 23 & 1.58 & - & - & - & 1.53 & 1.92 & 1.85 \\
\hline carseq24 & 3.35 & - & - & - & 3.37 & 3.63 & 3.27 \\
\hline carseq30 & - & - & 1774.50 & 1484.62 & - & - & - \\
\hline carseq31 & 1.70 & 71.91 & 0.48 & 0.18 & 1.71 & 2.09 & 1.95 \\
\hline carseq33 & 5.11 & 239.02 & 2.64 & 1.46 & 6.73 & 7.99 & 6.26 \\
\hline carseq34 & 3.75 & - & - & - & 3.79 & 4.20 & 3.94 \\
\hline carseq41 & 1.83 & 0.07 & 0.34 & 0.16 & 1.86 & 2.20 & 1.97 \\
\hline carseq43 & 4.69 & 137.48 & 28.75 & 12.78 & 4.83 & 5.46 & 5.24 \\
\hline carseq44 & 3.94 & - & - & - & 3.89 & 4.19 & 4.03 \\
\hline carseq48 & 4.04 & 0.46 & 3.88 & 3.07 & 4.14 & 4.12 & 4.29 \\
\hline carseq49 & 4.00 & 93.10 & 1928.90 & 659.18 & 4.01 & 4.60 & 4.28 \\
\hline carseq51 & 4.46 & 0.07 & 0.40 & 0.20 & 4.49 & 4.93 & 4.27 \\
\hline carseq53 & 4.35 & - & 0.58 & 0.35 & 4.90 & 4.71 & 4.64 \\
\hline carseq54 & 4.54 & 0.08 & 0.68 & 0.37 & 4.15 & 4.96 & 4.81 \\
\hline carseq55 & 4.46 & 0.06 & 0.40 & 0.23 & 4.13 & 4.41 & 4.70 \\
\hline carseq58 & 4.30 & 0.08 & 0.43 & 0.34 & 4.30 & 2.42 & 2.12 \\
\hline carseq59 & 4.15 & 0.06 & 0.35 & 0.23 & 4.23 & 4.53 & 4.41 \\
\hline carseq61 & 5.05 & - & - & & 5.19 & 5.40 & 5.26 \\
\hline carseq62 & 3.24 & 0.07 & 0.33 & 0.25 & 3.34 & 3.61 & 3.46 \\
\hline carseq63 & 4.98 & 1321.83 & 190.42 & 68.42 & 5.45 & 5.80 & 5.65 \\
\hline carseq65 & 5.07 & - & - & & 5.10 & 5.45 & 5.41 \\
\hline carseq67 & 3.08 & 0.12 & 1.25 & 0.61 & 3.72 & 3.52 & 2.93 \\
\hline carseq70 & 6.33 & 0.08 & 0.37 & 0.29 & 6.46 & 6.75 & 6.65 \\
\hline carseq72 & 202.28 & - & - & - & 213.92 & 180.55 & 154.64 \\
\hline carseq73 & 5.96 & - & - & - & 6.08 & 6.57 & 6.23 \\
\hline carseq74 & 5.92 & 0.06 & 0.50 & 0.33 & 6.02 & 6.26 & 6.24 \\
\hline carseq75 & 3.16 & 0.06 & 0.34 & 0.18 & 1.78 & 2.00 & 2.15 \\
\hline carseq76 & 7.03 & 2.02 & 4.38 & 2.38 & 7.00 & 7.50 & 7.30 \\
\hline carseq78 & 5.55 & 0.08 & 0.34 & 0.27 & 5.60 & 5.85 & 5.88 \\
\hline total solved & 43 & 24 & 35 & 35 & 43 & 45 & 45 \\
\hline
\end{tabular}

median speed-up of 11.3 times. Furthermore, there are 4 instances (namely, carseq10, carseq19, carseq20, and carseq30) that cannot be solved using IloSequence alone within a one hour time limit, while applying our algorithms cREG and GSa does allow solving them. The most striking examples are carseq19 and carseq20, which can now be solved in 0.18 and 1.35 seconds, respectively, by using the GSa algorithm. 
Table 4 gen-sequence constraint on sequences of varied lengths

\begin{tabular}{|c|c|c|c|c|c|c|}
\hline \multicolumn{3}{|c|}{ instance } & \multicolumn{2}{|c|}{ gcc's + sequence's } & \multicolumn{2}{|c|}{ gen-sequence } \\
\hline characteristics & size & \#solutions & BT & $\mathrm{CPU}$ & BT & $\mathrm{CPU}$ \\
\hline \multirow{5}{*}{$\max 6 / 8-\min 22 / 30$} & 40 & 2,284 & 185,287 & 216.49 & 0 & 0.77 \\
\hline & 50 & 4,575 & 186,408 & 369.12 & 0 & 2.09 \\
\hline & 60 & 6,567 & 188,242 & 621.99 & 0 & 3.60 \\
\hline & 70 & 2,810 & 195,697 & 840.52 & 0 & 1.88 \\
\hline & 80 & 730 & 198,091 & 1061.62 & 0 & 0.61 \\
\hline \multirow{5}{*}{$\max 6 / 9-\min 20 / 30$} & 40 & 3 & 393,748 & 390.93 & 0 & 0.01 \\
\hline & 50 & 3 & 393,748 & 660.74 & 0 & 0.02 \\
\hline & 60 & 3 & 393,748 & 1074.26 & 0 & 0.03 \\
\hline & 70 & 3 & 393,748 & 1432.20 & 0 & 0.04 \\
\hline & 80 & 3 & 393,748 & 1786.62 & 0 & 0.05 \\
\hline \multirow{5}{*}{$\max 7 / 9-\min 22 / 30$} & 40 & 137,593 & 328,376 & 417.63 & 0 & 34.43 \\
\hline & 50 & 388,726 & 456,937 & 1061.24 & 0 & 150.87 \\
\hline & 60 & 718,564 & 729,766 & 2822.09 & 0 & 339.89 \\
\hline & 70 & 105,618 & $1,743,518$ & 5048.84 & 0 & 60.82 \\
\hline & 80 & 22,650 & $1,847,335$ & 7457.36 & 0 & 15.41 \\
\hline
\end{tabular}

\subsection{Multiple Sequence Constraints of Varied Lengths}

Table 4 evaluates the performance of the gen-sequence constraint on three families of instances constructed so that they are challenging for a $\mathrm{CP}$ approach using previously known constraints. The instances specify restrictions for work/rest patterns in individual schedules of rostering problems, inspired by contexts in which several levels of time granularity (day, week, month, year) coexist [16]. Every instance requires between 4 and 5 days worked per calendar week. Its identification, of the form "maxA/B-minC/D", indicates that at most A days are worked in any B consecutive days but that at least $\mathrm{C}$ days are worked in any $\mathrm{D}$ consecutive days. Instance size, i.e., the number of days in the scheduling horizon, varies from 40 to 80 within each family. We wish to enumerate all the solutions. The "gcc's + sequence's" model uses one gcc constraint per week and two separate sequence constraints for the A/B and C/D restrictions. Note that the sequence constraints are implemented with our customized algorithm. The "gen-sequence" model uses a single gen-sequence constraint. Since we achieve domain consistency for this constraint and the whole problem is captured by this one constraint, the number of backtracks is always zero. These few experiments suffice to show that using the gen-sequence constraint in the general setting can lead to computational savings of several orders of magnitude.

\section{Discussion}

We proposed, analyzed, and experimentally evaluated three new filtering algorithms for the sequence constraint. They have different strengths that complement each other well. The local graph approach of Section 3 does not guarantee domain consistency but often results in a significant amount of filtering, as witnessed in the experiments. Its asymptotic time complexity is $\mathrm{O}\left(n q^{2}\right)$. The reformulation as a regular 
constraint, described in Section 4, establishes domain consistency but its asymptotic time and space complexity are exponential in $q$, namely $\mathrm{O}\left(n 2^{q}\right)$. Nevertheless it performs very well, partly due to its incremental nature, for small values of $q$, not uncommon in applications: in car sequencing, values between 2 and 5 are frequent; in rostering, the shift assignment problem typically features values between 5 and 9 whereas the shift construction problem may require values up to 12 . The customized algorithm of Section 5 also establishes domain consistency on the sequence constraint. It has an asymptotic time complexity that is polynomial in $n$, namely $\mathrm{O}\left(n^{3}\right)$ along each path from the root to a leaf in the search tree. Also in practice this algorithm performed very well, being often even faster than the local graph approach. It should be noted that previously known algorithms did not establish domain consistency.

In our experimental section, we demonstrated the advantages of our proposed algorithms in practice. On single sequence constraints with various parameters, our algorithms outperform the state of the art by several orders of magnitude. In more realistic settings such as the car sequencing problem, we showed that our algorithms allow solving more instances, or again can improve the state of the art significantly. Finally, on more complex combinations of among constraints, our generalized sequence algorithm achieves computational savings of orders of magnitude.

Our contribution extends beyond the sequence constraint and into more general combinations of among (-like) constraints. The algorithm of Section 5 also establishes domain consistency in $\mathrm{O}\left(n^{4}\right)$ time on freer combinations of among constraints, as long as each is defined on consecutive variables with respect to a fixed ordering. In this context, it is worth recalling that not every combination of among constraints is tractable-Régin proved that finding a solution to an arbitrary combination of among constraints is NP-complete [11]. Another interesting extension for our first two algorithms is that they lend themselves to a generalization of among in which the number of occurrences is represented by a set (as opposed to an interval of values).

Acknowledgements The authors would like to thank Marc Brisson and Sylvain Mouret for their help with some of the implementations and experiments.

A preliminary version of this article appeared at CP-06, the $12^{\text {th }}$ International Conference on Principles and Practice of Constraint Programming, Nantes, France in September 2006 [15]. This work was partially supported by the Canadian Natural Sciences and Engineering Research Council (Discovery grant OGP0218028), the Intelligent Information Systems Institute (IISI) at Cornell University (AFOSR grant FA9550-04-1-0151), and the Defense Advanced Research Projects Agency (DARPA; REAL grant FA8750-04-2-0216, COORDINATORs grant FA8750-05-C-0033).

\section{References}

1. K. Apt. Principles of Constraint Programming. Cambridge University Press, 2003.

2. N. Beldiceanu and M. Carlsson. Revisiting the Cardinality Operator and Introducing the CardinalityPath Constraint Family. In P. Codognet, editor, Proceedings of the 17th International Conference on Logic Programming (ICLP 2001), volume 2237 of LNCS, pages 59-73. Springer, 2001.

3. N. Beldiceanu and E. Contejean. Introducing global constraints in CHIP. Journal of Mathematical and Computer Modelling, 20(12):97-123, 1994.

4. N. Beldiceanu, M. Carlsson, and J.-X. Rampon. Global Constraint Catalog. Technical Report T200508, SICS, 2005. 
5. R. Dechter. Constraint Processing. Morgan Kaufmann, 2003.

6. S. Demassey, G. Pesant, and L.-M. Rousseau. A cost-regular based hybrid column generation approach. Constraints, 11:315-333, 2006.

7. M. Dincbas, H. Simonis, and P. van Hentenryck. Solving the car-sequencing problem in constraint logic programming. In Y. Kodratoff, editor, Proceedings of the European Conference on Artificial Intelligence (ECAI), pages 290-295, 1988.

8. I. Gent and T. Walsh. CSPLib: a benchmark library for constraints. Technical report, TR APES-091999, 1999. Available at http://www.csplib.org.

9. R. Mohr and G. Masini. Good Old Discrete Relaxation. In European Conference on Artificial Intelligence (ECAI), pages 651-656, 1988.

10. G. Pesant. A Regular Language Membership Constraint for Finite Sequences of Variables. In M. Wallace, editor, Proceedings of the Tenth International Conference on Principles and Practice of Constraint Programming (CP 2004), volume 3258 of Lecture Notes in Computer Science, pages 482-495. Springer, 2004.

11. J.-C. Régin. Combination of Among and Cardinality Constraints. In R. Barták and M. Milano, editors, Proceedings of the Second International Conference on Integration of AI and OR Techniques in Constraint Programming for Combinatorial Optimization Problems (CP-AI-OR 2005), volume 3524 of Lecture Notes in Computer Science, pages 288-303. Springer, 2005.

12. J.-C. Régin. Generalized Arc Consistency for Global Cardinality Constraint. In Proceedings of the Thirteenth National Conference on Artificial Intelligence and Eighth Innovative Applications of Artificial Intelligence Conference (AAAI / IAAI), volume 1, pages 209-215. AAAI Press / The MIT Press, 1996.

13. J.-C. Régin and J.-F. Puget. A Filtering Algorithm for Global Sequencing Constraints. In G. Smolka, editor, Proceedings of the Third International Conference on Principles and Practice of Constraint Programming (CP97), volume 1330 of LNCS, pages 32-46. Springer, 1997.

14. M. Trick. A Dynamic Programming Approach for Consistency and Propagation for Knapsack Constraints. Annals of Operations Research, 118:73-84, 2003.

15. W.-J. van Hoeve, G. Pesant, L.-M. Rousseau, and A. Sabharwal. Revisiting the sequence constraint. In CP-06: 12th International Conference on Principles and Practice of Constraint Programming, volume 4204 of Lecture Notes in Computer Science, pages 620-634, Nantes, France, Sept. 2006.

16. T. Zemmouri, P. Chan, M. Hiroux, and G. Weil. Multiple-level Models: an application to employee timetabling. In E. K. Burke and M. Trick, editors, Proceedings of the 5th International Conference on the Practice and Theory of Automated Timetabling (PATAT'04), pages 397-412, 2004. 